\title{
Optimizing the redesign of La Turballe port including long term shoreline morphodynamics
}

\section{Megan COOK ${ }^{1,3,4}$, Frédéric BOUCHETTE ${ }^{1,3}$, Bijan MOHAMMADI ${ }^{1,2,3}$, Léa SPRUNCK ${ }^{1,3}$, Nicolas FRAYSSE ${ }^{4}$}

\section{GEOSCIENCES-M, Université de Montpellier, CNRS, Montpellier, France.} megan.cook@umontpellier.fr ; frederic.bouchette@umontpellier.fr ; lea.sprunck@umontpellier.fr

2. IMAG, Université de Montpellier, CNRS, Montpellier, France. bijan.mohammadi@umontpellier.fr

3. GLADYS, Université de Montpellier, CNRS, Le Grau du Roi, France.

4. BRL Ingénierie, Nîmes, France, nicolas.fraysse@brl.fr

\begin{abstract}
:
We present a numerical study as part of the project by BRLi to redesign La Turballe port. The port is home to a wide array of maritime activities, including fishing, ship repair, touristic boat rides and the presence of a marina. The increase of these activities as well as the arrival of others result in the need of enlarging the existing port. In addition to increasing its exploitable surface, the objective is to also reduce the agitation of the waves within the port. This paper aims to present the research conducted in finding a new layout of the port using optimal control, which accompanies the more traditional engineering approach. In addition to finding a new configuration of La Turballe port meeting both criteria, we look into the long term effects of this new layout on the surrounding shoreline.
\end{abstract} Keywords: Defence structure, Optimal control, Coastal engineering, Morphodynamics.

\section{Introduction}

The port of La Turballe, situated in the North-West region of France will undergo a number of transformations with the aim of increasing the surface capacity of the port for the purpose of accommodating the rising number of industrial, recreational and fishing activities, as well as those associated with the arrival of an offshore wind farm in 2021. The objective of the development project is to transform the geometry of the port, while respecting the usual engineering constraints and reducing as much as possible the agitation of the sea within the port. The final solution must answer two possibly conflicting questions: (1) Which transformations lead to a decrease of the agitation of the water within the port? (2) Which transformations increase the size of the port in order to optimize its capacity in terms of services? Since the fulfilment of one criterion may impair the other, there is no trivial solution that meets both criteria. In addition, the number of geometric transformations is large. Looking for the best solution taking into account both criteria using classic engineering processes may require a large number of exploratory 


\section{Thème 4 - Ouvrages portuaires, offshore et de plaisance}

numerical simulations, forced by a large range of different weather and sea conditions. On that basis, we introduce an approach by optimal control.

\section{Setting}

In this section, we present the port of La Turballe and the possible transformations considered, as well as the forcing conditions considered in this study.

\subsection{Presentation of La Turballe port}

Figure 1 shows the La Turballe port in its initial state as well as possible transformations considered acceptable by the different actors of the project. Through classic engineering, it was established that the best course of action was through the installation of a jetty and a mole to produce a bottleneck effect at the entrance of the port. The question remains regarding the shape and dimensions of these two defence structures.

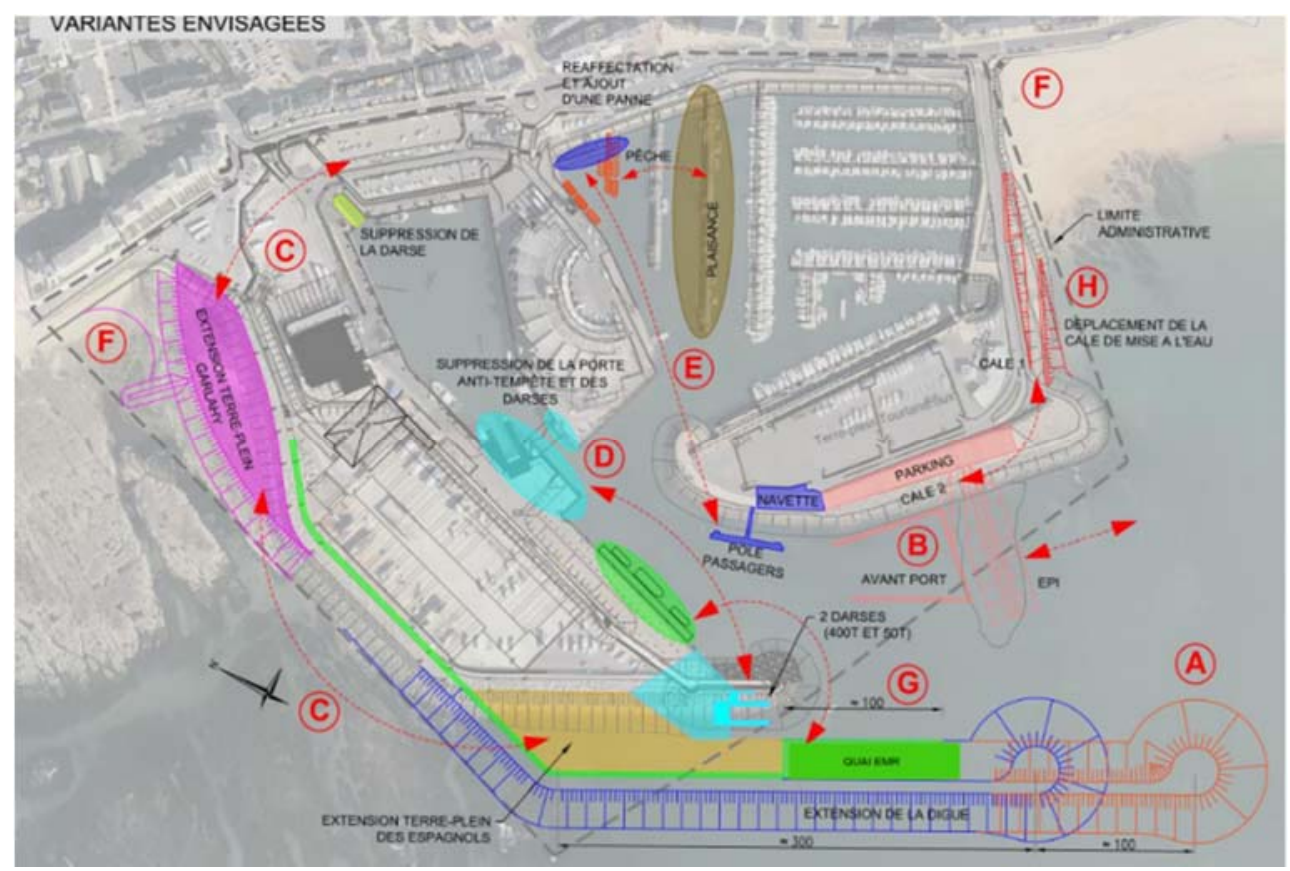

Figure 1. Development proposals established by a classical engineering approach and other constraints (socio-economic, legal, environmental) of the project. All the dimensions authorized on these arrangements determine the constraints imposed on the optimal calculation.

\subsection{Forcings}

Port agitation is directly controlled by ocean waves appearing at its entrance to the South with no other opening inside the port that could alter this wave field. We consider that the jetty can never be submerged and that no influx of energy can cross it. The hydrodynamic model considered can therefore be limited to the inside of the port, with a local forcing at 


\section{XVI'̀mes Journées Nationales Génie Côtier - Génie Civil \\ Le Havre 2020}

its entrance. Globeocean provided the forcing data in the form of different sea and weather conditions. This allowed us to define 28 different forcing scenarios representative of the conditions observed at La Turballe port. These scenarios are used in the search of an optimal port configuration.

\section{Methodology}

This section is devoted to the methods adopted in this study, which include a description of the concept of optimal control, its application to La Turballe port, and an outline of the hydrodynamic model used to describe the state of the water within the port.

\subsection{Optimal control}

The term optimization in coastal dynamics refers to the transformation of the natural seabed or the geometric and rheological properties of artificial structures present in ports or at the coast that leads to the minimization of a scalar quantity $\mathcal{J}$. This quantity, named cost function, is representative of the state of the system and is generally associated with certain physical quantities, such as those related to waves or currents. The general framework of optimal control in coastal zones is summarized in figure 2 where the domain $\Omega$ corresponds to a coastal zone or port. This approach to coastal management has been applied in various operations and has proved effective. We find a general presentation of the methods used here in: IVORRA et al., 2005; MOHAMMADI \& PIRONNEAU, 2001; MOHAMMADI \& SAIAC, 2003.

\subsection{Cost function}

For a given forcing scenario, we consider the following cost function as wave agitation:

$$
\mathcal{J}_{i}(\psi)=\frac{1}{K(\mathcal{P})} \int_{\Omega} \mathcal{E}_{i}(\psi, x) \cdot \mathcal{P}(x) \cdot d x
$$

where the index $i$ refers to a given forcing scenario and $\psi$ the parametrization of the port modifiable in the search of an optimal configuration; The quantity

$$
\mathcal{E}_{i}(\psi, x)=\frac{1}{2} \rho g \cdot\left(a_{i}(\psi, x)\right)^{2}
$$

is the total surface energy defined over the domain $\Omega$. Here, $\rho$ is the density of the water, $\mathrm{g}$ is the gravitational acceleration and $a_{i}(\psi)$ is the amplitude of the waves. The function $P$ enables us to prioritize the minimization of the agitation over certain preferred zones of the port, with $K(\mathcal{P})=\int_{\Omega} \mathcal{P}(x) d x$. In order to combine the 28 different forcing scenarios, we need to weight the cost functions $\mathcal{J}_{i}$ to form a global cost function $\mathcal{J}$. Since the search of an optimal configuration concerns the minimization of port agitation caused by the most energetic scenarios, it is natural to weight the cost functions based on the agitation forcing the system at the entrance of the port. As such, the global cost function to be minimized in the search of the optimal solution is defined as: 


\section{Thème 4 - Ouvrages portuaires, offshore et de plaisance}

$$
\mathcal{J}(\psi)=\frac{\sum_{i=1}^{28} a_{i}(\psi) \mathcal{J}_{i}(\psi)}{\sum_{i=1}^{28} a_{i}(\psi)}
$$

The following hydrodynamic model is needed to calculate the agitation $a_{i}(\psi)$ for each of the 28 scenarios and a given port configuration $\psi$.

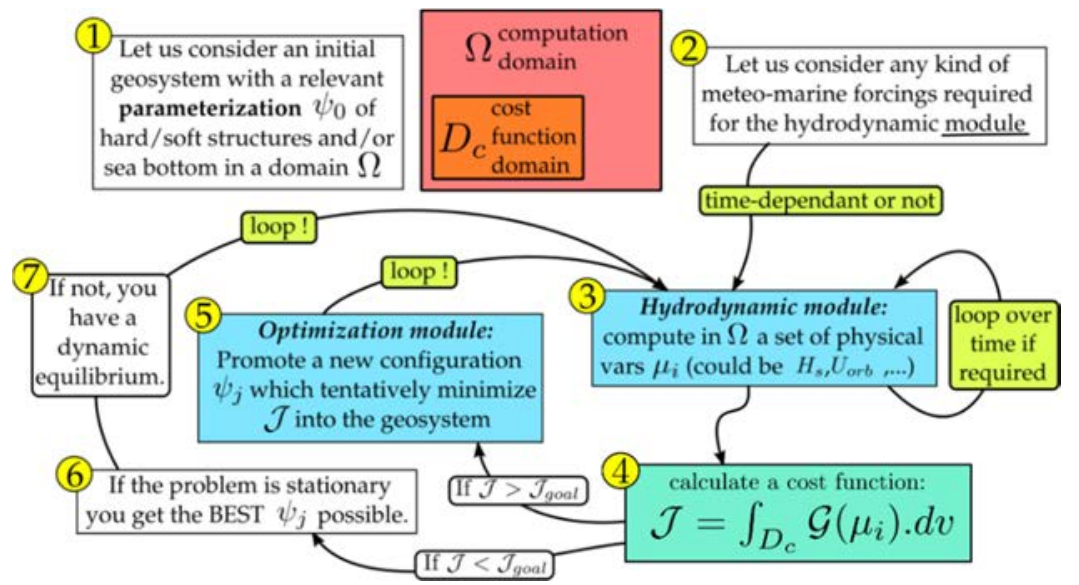

Figure 2. The concept of shape optimization applied to coastal engineering and coastal hydro-morphodynamics.

\subsection{Hydrodynamic model}

In the context of the port of La Turballe and given the forcing data provided by Globeocean, the most suitable principle describing the state of agitation dominated by seiches in the port is based on a form of the wave equation. A wave is considered as the sum of an incident wave and a reflected wave, where the reflected wave satisfies the Helmholtz equation with variable bathymetry (BERKHOFF, 1972; RADDER, 1979).

\section{Results}

We search for the optimal configuration of the port using two degrees of freedom: the length of the jetty and the shape/position of the mole. Two strategies were developed, addressing different needs. In both cases, the length of the jetty is questioned but the first case focuses on the size of the mole for the development of parking facilities whereas, the second prioritizes the size of the area accommodating waterborne vessels. The decision between the two depends on social, financial and political criteria. As such, we conducted both studies independently.

\subsection{Parking facility configuration}

The first study concerns the dimensioning of the port under the assumption that a mole capable of accommodating parking facilities is preferred. In the search of an optimal solution, we define the set of parameters by $\psi=(\alpha, \beta)$, where $\alpha$ is the width added to the mole $\mathrm{A}$ and $\beta$ is the length of the extension of the jetty $\mathrm{B}$, as illustrated by figure 3 . 


\section{XVİ̀mes Journées Nationales Génie Côtier - Génie Civil \\ Le Havre 2020}

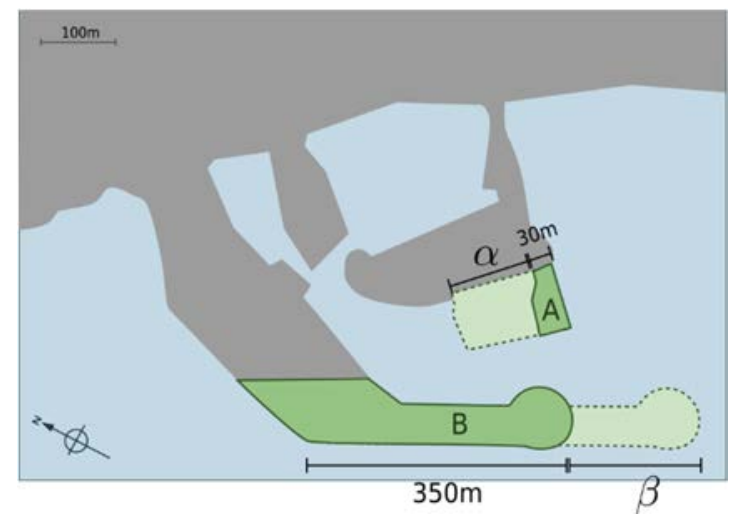

Figure 3. Illustration of the two degrees of freedom $\alpha$ and $\beta$.

The choice of these parameters originate from the expertise of classic port engineering, and incorporates the constraints mentioned in section 2.1. Figure 4 represents the values of the cost function $\mathcal{J}(3)$ with respect to the parameters $\alpha$ and $\beta$. The minimum of $\mathcal{J}$ is marked by a cross and is located away from the borders. We deduce that the study of the optimal solution with two degrees of freedom provides the following result: $\alpha=90$ and $\beta$ $=120$. The corresponding configuration is given in figure 5 . The jetty is extended by 120 $\mathrm{m}$ and the mole is widened by $90 \mathrm{~m}$.

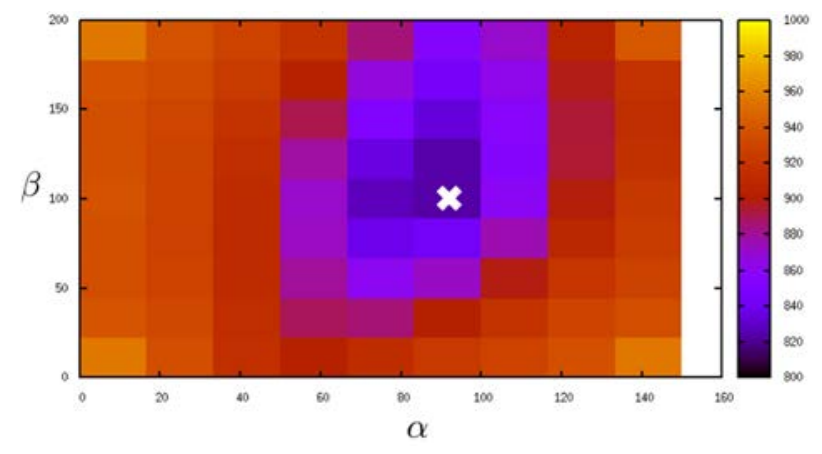

Figure 4. The cost function $\mathcal{J}(\alpha, \beta)$. The optimal solution is indicated by a cross.

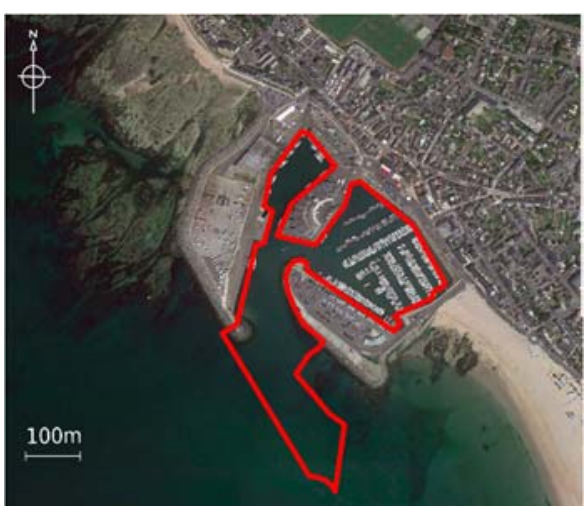

Figure 5. The port configuration corresponding to the cross situation.

\subsection{Boat harbor configuration}

The second study concerns the dimensioning of the port, under the assumption that a boat harbour is preferred. A mole will still be added to the developing structures but has a fixed shape and size. In this study, its position along the existing wall is considered. We define a different set of parameters $\psi=(\beta, \gamma)$. Here, $\beta$ remains the length added to the jetty and $\gamma$ defines the position of the mole along the existing wall. Figure 6 shows the values of the cost function (3) with respect to $\beta$ and $\gamma$, the minimum of which is marked by a cross. We deduce that the optimal solution with two degrees of freedom is given by: 


\section{Thème 4 - Ouvrages portuaires, offshore et de plaisance}

$\beta=0$ and $\gamma=91$. The corresponding configuration is given in figure 7 . The jetty measures $350 \mathrm{~m}$ and the mole is situated at $91 \mathrm{~m}$ from its initial position.

Figure 6 shows a local and a global minimum. This suggests that caution must be taken when performing the minimization, especially if we increase the number of design parameters, as new local minima may appear. In other words, in higher dimensions, optimization methods with global search capacity are of uttermost importance.

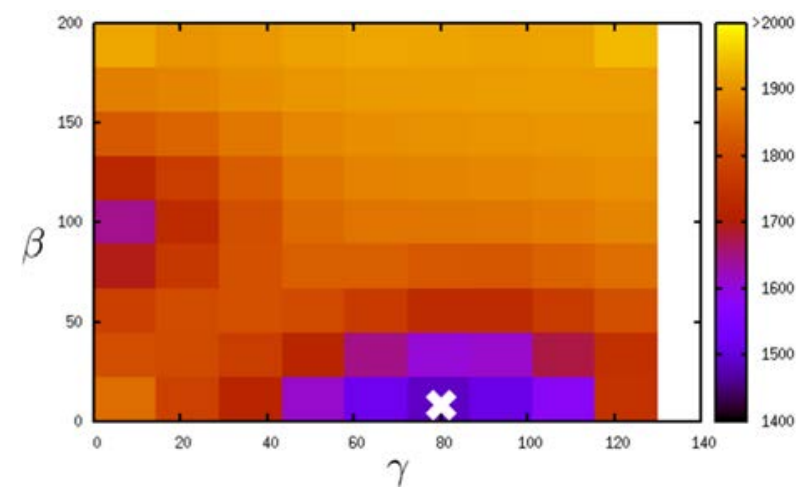

Figure 6. The cost function $\mathcal{J}(\beta, \gamma)$. The optimal solution is indicated by a cross.

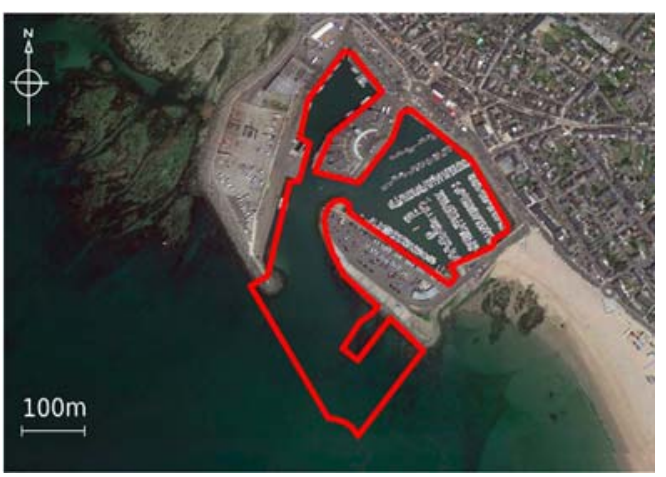

Figure 7. The port configuration corresponding to the cross situation.

\section{Discussion}

In this section, we discuss the two previously described optimal configurations as well as the morphological impact the additional structures have on the surrounding shoreline.

\subsection{Compare studies}

The study cases required by the main actors of the development plan possess two degrees of freedom. We compared the two solutions in order to determine the optimal configuration in terms of reducing wave agitation. Figure 8 compares the energy of the two optimal configurations presented previously. The global energy inside the port is calculated for both optimal configurations and forced by the 28 different scenarios. We observe that for most cases, the green line which corresponds to the energy associated with the parameters $(\alpha ; \beta)$ is above the red line, which corresponds to the energy associated with $(\beta ; \gamma)$. This study suggests that the optimal solution is the configuration prioritizing a boat harbor. We can therefore state that a shorter jetty and a better placed mole leads to a decrease of the global energy of the port. 


\section{XVİ̀mes Journées Nationales Génie Côtier - Génie Civil \\ Le Havre 2020}

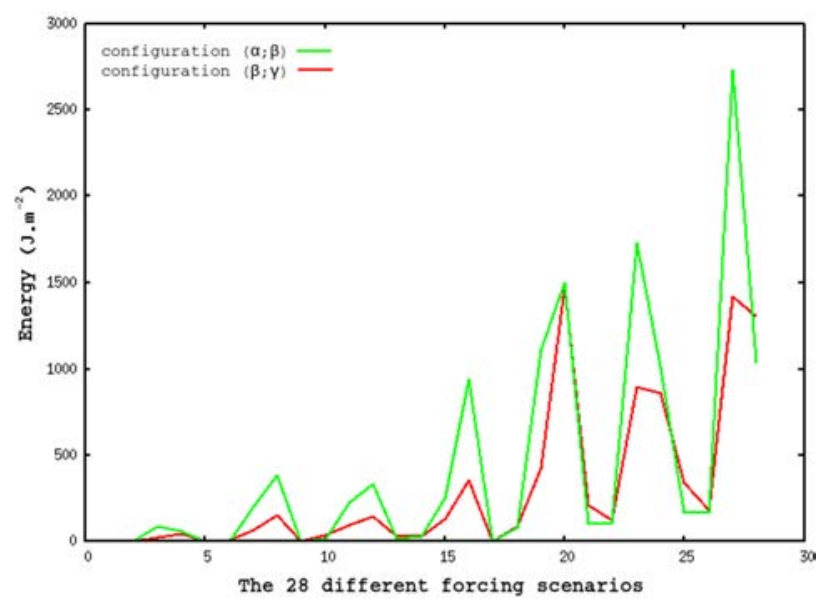

Figure 8. Comparison between the best configuration $(\alpha ; \beta)$ and the best configuration $(\beta ; \gamma)$.

\subsection{Morphodynamic study}

Given the previous study, an optimal solution to reduce the agitation within the port is to extend the jetty and modify the entrance of the port. As such, the question of the impact of these transformations on the long-term morphodynamics of the adjacent beaches arises. A numerical study was conducted on the long-term evolution of the coastline to verify that these modifications to the port do not have a significant impact on adjacent coastal sedimentary systems. Here, we present the results of the morphodynamic study applied to the optimal configuration presented in 4.1.

Two reference shorelines were defined as in figure 9a and synthetic forcings were exerted onto both systems over a period of 30 years. Figure $9 \mathrm{~b}$ shows the comparison of the two simulations, with and without the predicted port transformations, as well as the two initial shorelines. We notice that beyond $150 \mathrm{~m}$ on either side of the port, the shorelines with and without the transformations superimpose the original ones, which indicates no longterm disturbance of the shoreline by the new port configuration. We can conclude that the predicted reconfiguration of the port does not significantly impact the surrounding shoreline dynamics. A second study regarding the optimal $(\beta, \gamma)$ configuration yields the same conclusion.

\section{Conclusion}

Applying an optimal control method to the problem of the La Turballe port design leads to the conclusion that several configurations can be deemed optimal, depending on the parametrization of the jetty and mole. In terms of wave reducing efficiency, the different configurations are relatively equivalent. The decision on which one to adopt depends on social, financial and political criteria. Numerical simulations indicate that a reconfiguration of La Turballe port does not significantly impact the surrounding shoreline dynamics. 


\section{Thème 4 - Ouvrages portuaires, offshore et de plaisance}
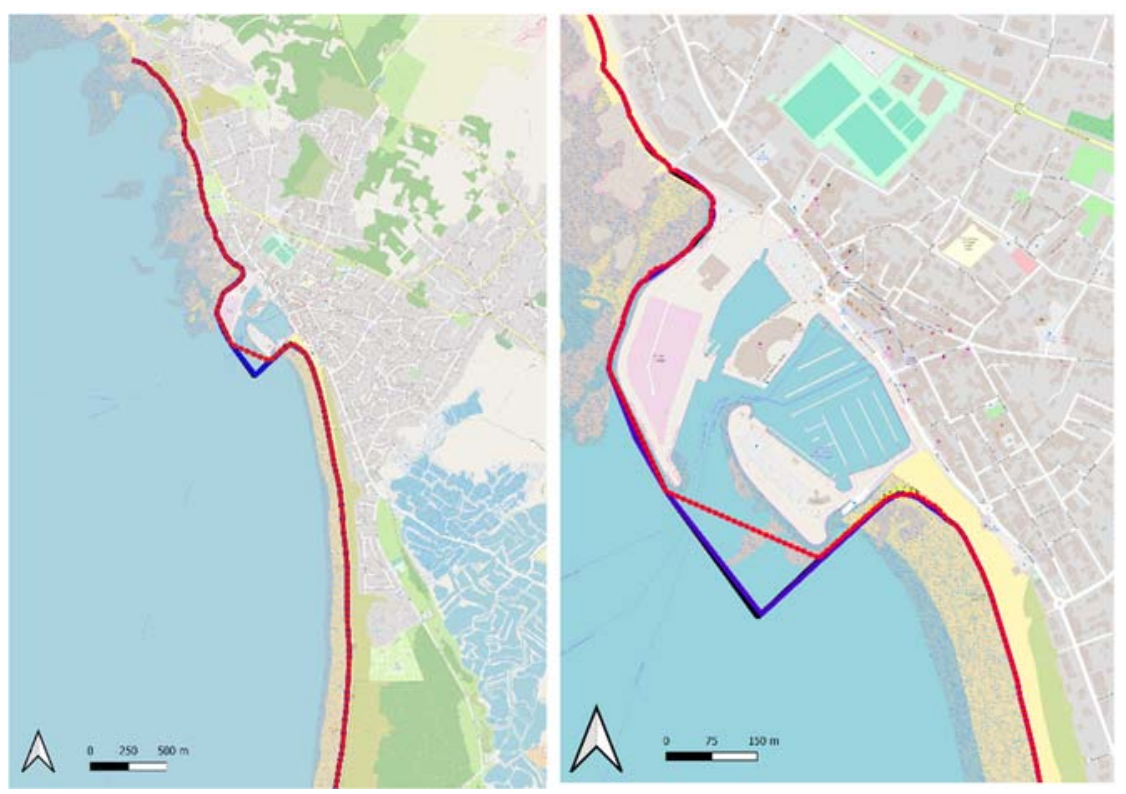

Figure 9. (a) Definition of the two reference shorelines. The red line represents the shoreline in its existing state (without the added structures) and the blue line represents the shoreline incorporating the extended jetty and widened mole.

(b) Results of the long term morphodynamic simulations with and without the proposed structures. The simulations without the redesign of the port are given by the dotted lines: yellow is the initial shoreline and red is the shoreline after the 30-year simulation. The simulations incorporating the redesign of the port are given by the solid lines: black is the initial shoreline and blue is the shoreline after the 30-year simulation.

Acknowledgment: This work is part of the $\mathrm{PhD}$ thesis work of Megan Cook, supported by BRLi. The authors would also like to acknowledge the financial and logistical support from the group GLADYS (www.gladys-littoral.org).

\section{References}

BERKHOFF J.C.W. (1972). Computation of combined refraction-diffraction. Proc. $13^{\text {th }}$ Coastal Eng. Conf., Vancouver, pp 471-490. https://doi.org/10.1061/9780872620490.027

IVORRA B., ISÈBE D., MOHAMMADI B. (2005). Optimisation globale à complexité

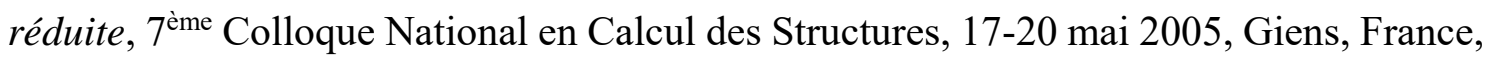
652 p., Hermes-Lavoisier (Ed.).

MOHAMMADI B., PIRONNEAU O. (2001). Applied shape optimisation for fluids. Oxford University Press, $251 \mathrm{p}$.

MOHAMMADI B., SAIAC J.H. (2003). Pratique de la simulation numérique. Dunod, $438 \mathrm{p}$.

RADDER A.C. (1979). On the parabolic equation method for water-wave propagation. Journal of Fluid Mechanics, Vol. 95(1), pp 159-176. https://doi.org/10.1017/S0022112079001397 